\title{
Editorial
}

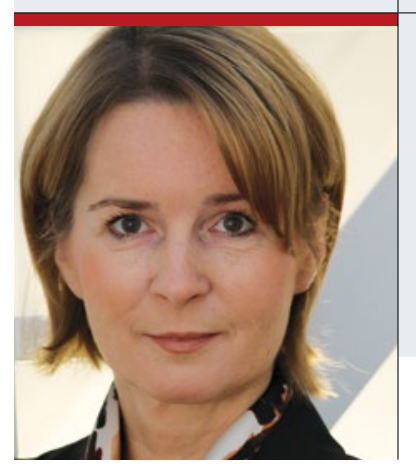

Katja Kupfer-Geißler

Chefredaktion

katja.kupfer@springer.com

facebook.com/springerpflege

\section{Gut beraten}

$\mathrm{K}$ ranke Kinder bedeuten ja in den meisten Fällen für die besorgten Eltern „nur“ ein paar durchwachte Nächte. Was aber, wenn man ein schwerstpflegebedürftiges Kind daheim hat, das dauerhaft Pflege braucht, das zuhause langzeitbeatmet wird oder das auf eine enterale oder parenterale Ernährung angewiesen ist? In einer solchen Situation sind Eltern genau so gefordert wie die professionelle Pflege. In einem speziellen PflegeKolleg für die ambulante Pflege (ab Seite 9) haben wir nicht nur die Themen Langzeitbeatmung und Ernährung aufgegriffen, sondern auch den nicht zu unterschätzenden Aspekt der Elternberatung. Denn mit einer Pflegeberatung gemäß $\$ 45$ SGB XI können Pflegekräfte die häusliche Pflegesituation deutlich stabilisieren und den Eltern Sicherheit und Unterstützung vermitteln. Als Pflegekraft, so schreibt unser Autor Tobias Brentle, können Sie entscheidend dazu beitragen, die Lebensqualität des Kindes zu steigern, Ängste abzubauen und eine Normalisierung des Alltags zu erreichen.
Seit zwei Jahren gibt es in Hameln ein Demenzdorf, das erste - und bislang einzige - in Deutschland. Woran liegt es, wenn dort doch alle Plätze besetzt sind und die Warteliste lang ist, dass sich noch kein Nachahmer gefunden hat? Den Einblick ins Dorfleben verschafft uns Jennifer Minke-Beil ab Seite 46.

Und jetzt bleibt mir nur noch, Ihnen im Namen der gesamten Redaktion einen schönen Sommer zu wünschen!

Ihre

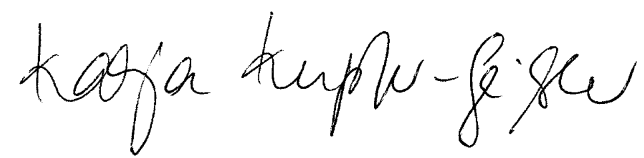

HEILBERUFE erscheint in diesem Monat mit einer Doppelnummer - die nächste Ausgabe erhalten Sie daher am 5 . September!

\section{GESUNDHEITSPFLEGE-KONGRESSS}

\section{Einladung nach Hamburg}

Am 4. und 5. November 2016 findet im Radisson Blu Hotel in Hamburg der 14. Gesundheitspflege-Kongress von Springer Pflege statt. Auf dem Pflegegipfel des Nordens werden wieder rund 800 Pflegefachkräfte, Stations- und Pflegedienstleitungen, PflegedirektorInnen, Lehrer, Lehrende und Studenten erwartet. Freuen Sie sich auf den Austausch mit Kollegen und ein aktuelles Fortbildungsprogramm, das Springer Pflege in bewährter Weise mit seinen Kooperationspartnern zusammenstellt. Schwerpunkte sind u.a. Patientenorientierung, Pflege- und Ergebnisqualität, Auswirkungen des neuen Pflegeberufsgesetzes, der Arbeitszeitgestaltung und die Schulung von Patienten und Angehörigen.

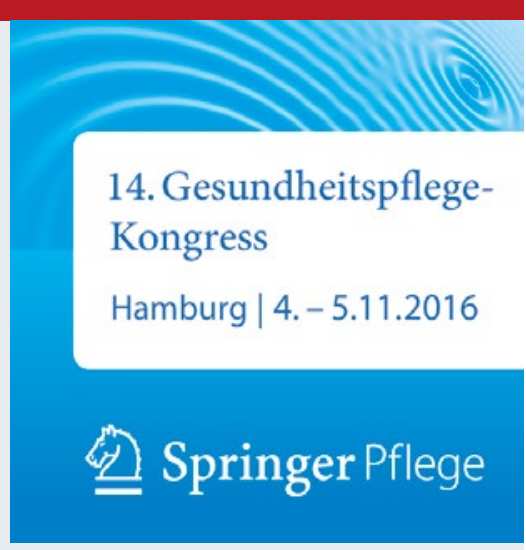

www.gesundheitskongresse.de 